\title{
Medulloblastoma: Molecular Classification-Based Personal Therapeutics
}

\author{
Tenley C. Archer ${ }^{1,2} \cdot$ Elizabeth L. Mahoney ${ }^{1} \cdot$ Scott L. Pomeroy ${ }^{1,2}$
}

Published online: 6 April 2017

(C) The American Society for Experimental NeuroTherapeutics, Inc. 2017

\begin{abstract}
Recent advances in cancer genomics have revealed 4 distinct subgroups of medulloblastomas, each with unique transcription profiles, DNA alterations and clinical outcome. Molecular classification of medulloblastomas improves predictions of clinical outcome, allowing more accurate matching of intensity of conventional treatments with chemotherapy and radiation to overall prognosis and setting the stage for the introduction of targeted therapies.
\end{abstract}

Keywords Medulloblastoma $\cdot \mathrm{WHO} \cdot \mathrm{MYC} \cdot \mathrm{SHH} \cdot \mathrm{WNT} \cdot$ Genomic $\cdot$ DNA methylation $\cdot$ Subgroups $\cdot$ Group $3 \cdot$ Group 4

\section{Introduction}

Medulloblastoma is one of the most common malignant brain cancers in children and accounts for $8 \%$ to $10 \%$ of childhood brain tumors. Medulloblastoma research has also been on the leading edge of cancer genomics. The insights gained from genome-wide analysis of DNA alterations and gene transcription are starting to translate to the clinic. These studies have shown that medulloblastomas consist of at least 4 distinct subgroups that have unique transcription profiles, somatic DNA alterations, and clinical outcomes. An international consensus defining these subgroups has been accepted as the basis for diagnostic criteria by the World Health Organization (WHO), revealed in the recently published revised fourth

Scott L. Pomeroy

scott.pomeroy@ childrens.harvard.edu

1 Department of Neurology, Boston Children's Hospital, Boston, MA 02115, USA

2 Harvard Medical School, Boston, MA 02115, USA edition of the WHO Classification of Tumors of the Central Nervous System [1, 2]. The WHO classification now includes medulloblastoma molecular subgroups that are of immediate prognostic value for patients, and set the stage for clinical trials of new targeted therapies (Table 1).

We and others first profiled the genomic mutation landscape of medulloblastoma in 2012 [3-5]. Three papers published back-to-back identified mutated genes, and delineated the extent to which these mutations were specific to the transcription- and copy number-based subgroups previously characterized [1, 6-9].

Now, nearly 5 years later, these findings are just starting to translate to clinical trials [10-14]. Standardization of the methods to define medulloblastoma subgroups in a "realtime" clinical setting remains a significant challenge, but the WHO guidelines will help to pull subgroup methods from the laboratory into the clinic more rapidly (Table 1). Accurate and timely clinical subtype assignments will be the basis for molecular subclassification-based personal therapeutics in medulloblastoma. With tumor subgroup in hand, it will plausible to more precisely risk-stratify patients and tune traditional treatment modalities, and identify candidates for emerging targeted therapies.

Medulloblastoma research is now moving into the postgenomic era of cancer research. Most cancer types have had large cohort sequencing studies published, and it is likely that the large majority of coding sequence oncogenic mutations have been discovered $[15,16]$. Practical implementation of genomic methods is feasible owing to the plummeting cost from evolving DNA sequencing technologies [17]. However, knowing the population-wide genetic events in cancer has not yielded a corresponding systemic shift in survival rates.

Historically, the largest major leap forward in cancer survival for most solid tumors came in the $1950 \mathrm{~s}$ - when radiation treatment became part of the standard of care for most 
Table 12016 World Health Organization (WHO)

Histological Classification of Tumours of the Central Nervous System - medulloblastoma diagnosis (modified from [2])

\begin{tabular}{lll}
\hline WHO classification subgroup & Histology subtype & Prognosis and demographic notes \\
\hline Medulloblastoma, WNT-activated & Classic & Low risk \\
& $\begin{array}{l}\text { Large cell/anaplastic } \\
\text { (very rare) }\end{array}$ & Uncertain clinicopathological significance \\
Medulloblastoma, SHH-activated, & Classic (rare) & High risk \\
TP53 mutant & Large cell/anaplastic & High risk; prevalent in children \\
& & aged 7-17 y \\
& Desmoplastic/nodular & Uncertain clinicopathological significance \\
Medulloblastoma, SHH-activated, & Classic & Standard risk \\
TP53 wild type & Large cell/anaplastic & Uncertain clinicopathological significance \\
& Desmoplastic/nodular & Low risk in infants; prevalent in \\
& & infants and adults \\
Medulloblastoma, & Extensive nodularity & Low risk in infants \\
non-WNT/non-SHH, group 3 & Classic & Standard risk \\
Medulloblastoma, & Classic & High risk \\
non-WNT/non-SHH, group 4 & Large cell/anaplastic (rare) & Uncertain clinicopathological significance \\
\hline
\end{tabular}

WNT $=$ wingless; $\mathrm{SHH}=$ Sonic hedgehog cancers [18]. This was owing to the discovery of megavoltage linear accelerators (linac) machines. Linacs delivered radiation with more precision, at higher dosages, and with deeper penetrance than the previous technology [19]. Since the 1970s, the 5-year overall survival rate for all patients with cancer has increased by about 20\% (American Cancer Society Cancer Facts and Figures 2016). These gains came from early diagnosis, and chemotherapy treatments. Some cancers, like prostate, testicular, and leukemias, have seen the biggest gains. Survival rates of other cancers have barely changed, such as lung cancer survival rates, which have only increased $3 \%$ to $16 \%$ [20].

There are 2 main reasons that have constrained cancer genomic insights from translating to improved therapies. First, as is the case for medulloblastoma, there are few druggable targets, in part owing to a lack of effective drugs, and also because of the striking heterogeneity of genomic alterations across patient tumors samples even within a subgroup. Second has been the instability of the disease with continued acquisition of new genetic abnormalities, which allows for rapid development of resistance to targeted therapies. Indeed, even for cancers with a large percentage of patients having the same genetic alteration that a targeted therapy can be designed against - V600E BRAF mutations in melanoma being an example - the tumors evolve rapid resistance to that targeted therapy vemurafenib (PLX4032) [21-23]. A clear exception to this is leukemia-specifically chronic myeloid leukemia (CML). CML proves that the right targeted therapy can restore normal life expectancy [24]. Close to $90 \%$ of all CMLs share the same BCR-ABL fusion, or so-called Philadelphia chromosome. The tip of the long arm of chromosome 9 breaks at the ABL gene and this becomes fused to another break in chromosome 22 at the BCR gene. This creates a novel oncoprotein $\mathrm{BCR}-\mathrm{ABL}$ which functions to maintain constitutive tyrosine kinase activity. The tyrosine kinase inhibitor imatinib (Gleevec) inhibits phosphorylation of the BCR-ABL target [25]. Gleevec prevents the progression of CML into the fatal form, but it must be taken continuously [26]. As with other cancers, emergence of resistance in responsive tumors has been an issue, but further development of targeted therapies has enabled therapists to overcome this resistance in many cases. The success of Gleevec demonstrates that a well-characterized genetic lesion that is stable from patient to patient makes it a tractable system to find the best drug.

More heterogeneous cancers, like medulloblatoma, are going to require a new model for drug discovery that is based upon more of a personalized medicine approach, or at least a subgroup-focused approach based on within subgroup similarities. Subgroups based on histology were first reported in the $1980 \mathrm{~s}$, but they were found to predict poorly clinical behavior [27, 28]. In 2002, the analysis of genome-wide arraybased expression data demonstrated that biologically distinct molecular subgroups exist and that gene expression profiles were more accurate for predicting clinical behavior than histologic or clinical criteria [9]. Further refinement of methods and increased tumor cohort size has led to the current classification schema, as noted above. Subgroup-specific approaches are being adopted for clinical trials, allowing modification of standard treatments, including changes in radiation dosage or chemotherapy schedules, and the inclusion of novel targeted interventions. Now is the time that we can start to increase survival rates that have held steady for nearly half a century while at the same time improve the quality of life of survivors by reduced toxicity of treatment, and this will be 
done by finding the right treatments for the right group of patients.

\section{Medulloblastoma Subgroups and Clinical Significance}

Medulloblastoma comprises multiple molecular subgroups, which, importantly, have distinct prognoses [1, 6-9]. There is an international consensus that 4 major subgroups exist, and their names reflect our understanding of their mechanismsWNT, SHH, group 3, and group 4 [1, 6, 29], but as this consensus was reached it was already clear that there are molecular subgroups within these subgroups that have clinical relevance, which was clearly reinforced in a retrospective meta-analysis of 402 tumors $[6,30]$. These subgroups are durable and further recapitulated using other genome-wide data such as DNA methylation profiles [31].

Current treatment regimens for medulloblastoma patients have stayed largely consistent for the last 2 decades. For children $>3$ years of age, it is based on external beam radiation to the brain and spine with a boost to the tumor bed, together with combinations of multidrug chemotherapy [32, 33]. Medulloblastoma has 2 main treatment stratification levels based on clinical criteria - average risk and high risk [34]. Average-risk medulloblastomas have $<1.5 \mathrm{~cm}^{2}$ residual tumor following surgical resection and no metastases, while high risk are metastatic at diagnosis and/or $>1.5 \mathrm{~cm}^{2}$ residual tumor [35]. Average-risk tumors are treated with 23.4 Gy craniospinal irradiation, with a boost to the tumor bed of 55.8 Gy. Higher risk increases the craniospinal irradiation radiation to between 36.0 and $39.6 \mathrm{~Gy}$, depending on extent of metastasis. Both average and high-risk patients receive a multidrug chemotherapy regime of four 28-day cycles of cyclophosphamide, cisplatin, and vincristine [34, 36, 37]. Children $<3$ years of age are treated are differently. For these children, a major goal is the elimination or great reduction of radiation to the brain and spine, which have been shown to be particularly devastating to the developing brain in these early years of life. Indeed, high-dose chemotherapy in young children and infants without radiation gives nearly $75 \%$ overall 5-year survival for favorable subgroups such as the desmoplastic nodular variant [38].

This treatment scheme has led to excellent overall survival rates in medulloblastoma- $85 \%$ overall 5-year survival for average risk and $70 \%$ for high risk [11, 39]. Unfortunately, these treatments can be devastating to the survivors. Surgery itself has many complications such as cerebellar mutism syndrome, which causes detrimental effects on speech, cognition, and behavior of the patients [40]. Radiation is even more devastating to the quality of life in the survivors [13, 41]. Medulloblastoma is generally more radiosensitive than other brain cancers such as glioblastoma, but radiation dosages have not been able to be further reduced without negatively affecting survival [42-44]. Radiation reduces IQ and the magnitude of the reduction is inversely related to the age of the patients. The youngest patients can lose up to 40 IQ points [45]. Many of these children do not grow up to live independently as adults [41, 46, 47]. Radiation can cause secondary cancers, hearing loss, and radiation-induced vasculopathy and strokes [48-51]. There is a desperate need for improved therapies in medulloblastoma that could help to reduce the amount of radiation needed, and especially in the youngest of patients.

Central to the goal of reducing radiation is to identify improved targeted therapies in medulloblastoma. Targeted therapies are likely to be predicated on medulloblastoma subgroups. Having the 2016 WHO guidelines for medulloblastoma diagnosis incorporate subgroups will be a major leap forward toward these goals (Table 1). Here we list the WHO subgroups, give their defining features from molecular and genomic studies and summarize the current therapeutic opportunities these may have.

\section{Wingless Activated}

Wingless (WNT) signaling activated subgroup tumors are the least common of the medulloblastoma subgroups, accounting for just $11 \%$. Overall, medulloblastoma afflicts boys more often than girls, but the WNT subgroup affects both sexes equally. The peak age of diagnosis for WNT tumors is 6 to 10 years of age [52]. WNT is also remarkable for its very favorable prognosis. Nearly $90 \%$ of patients survive for more than 5 years [53]. WNT tumors are named because they are dominated by a WNT signaling and activated $\beta$-catenin (CTNNB1) signature. Indeed, the DNA sequencing studies identified that almost every tumor in the subgroup harbors a stabilizing point mutation in CTNNB1 [4, 5]. The majority of WNT subgroup tumors share similar genomic events; in addition to $C T N N B 1$, almost universally they have monosomy chromosome 6 . The WNT activated subgroup can be clinically diagnosed by nuclear accumulation of catenin beta 1 (CTNNB1) immunohistochemistry of tumor sections [54]. Furthermore, half of all WNT tumors contain mutations in X-linked RNA helicase $D D X 3 X$. $D D X 3 X$ has subsequently been found in to be recurrently mutated in a number of other cancers, including head-and-neck, mesothelioma lung cancer, lymphoma, and leukemia [55-59]. As seen in medulloblastoma, $D D X 3 X$ is on the $\mathrm{X}$ chromosome and it appears to have also have a strong sex bias for mutations $[60,61]$.

Germline mutations can be associated with WNT medulloblastoma, but these are rare events. A small cohort study, including $10 \mathrm{WNT}$ tumors, identified 2 patients with germline lesions in anaplastic lymphoma kinase gene $(A L K)$, a tyrosine kinase receptor, [62]. A separate case study had previously reported a germline $A L K$ mutation in a medulloblastoma 
tumor of unknown subgroup [63]. ALK mutations lead to constitutive activation of nuclear factor kappa B pathway, which may indirectly activate WNT through crosstalk of the pathways $[64,65]$. Germline mutations in another WNT pathway regulator can also lead to medulloblastoma. Turcot syndrome with familial adenomatous polyposis is an inherited cancer predisposition syndrome that is caused by loss-of-function mutations in adenomatous polyposis coli (APC) [66]. Familial adenomatous polyposis is associated both with colorectal adenomas and medulloblastoma. APC downregulates $\beta$-catenin directly through phosphorylation events; consequently, its mutations lead to increased WNT signaling [67]. Somatic mutations in APC have also been identified in spontaneous medulloblastoma [68].

Recent work has suggested that the favorable prognosis in WNT tumors is because they have a very leaky vasculature, which allows for improved delivery of chemotherapy agents to the tumor cells [69]. The breakdown in the blood-brain barrier in WNT tumors is thought to be because they secrete the diffusible WNT antagonists, WIF1 and Dickkopf-related protein 1 . Inhibiting WNT signaling to the surrounding vasculature disrupts its integrity as WNT is required for proper angiogenesis in the central nervous system [70]. Clinical trials are currently underway to leverage these insights so that patients with WNT subgroup medulloblastomas can be effectively treated with less chemotherapy and/or less radiation (ClinicalTrials.gov identifiers NCT01878617 and NCT02212574). There is some preliminary evidence to support that patients with WNT tumors treated with less radiation do, indeed, preserve more of their intellectual capabilities [13].

Drugging the WNT pathway for treatment of cancers, not just medulloblastoma, has been a vexing problem [71, 72]. WNT signaling is used so ubiquitously for key functions in the human body that its inhibition is not without risks and side effects. For example, it is required for bone formation and its reduction can lead to osteoporosis. Furthermore, the signal transduction pathway has so many multilayered points of regulation it is not always clear what level in the pathway is best to target for precise and durable benefit. As WNT-activated medulloblastoma tumors are nearly universally driven by stabilizing mutations in $C T N N B 1$, targeting its interaction with another transcription factor, CREB binding protein (CREBBP), to inhibit transcription of their target genes would be a rational approach. PRI-724 is a CREBBP:CTNNB1 interaction antagonist that is being investigated in phase I clinical trials in pancreatic and liver cancers (ClinicalTrials.gov identifier NCT02413853).

In medulloblastoma, drugging the WNT pathway will need to be done with caution. It is worth noting that WNT medulloblastoma tumors can also harbor mutations in CREBBP, and the extent to which this may affect the efficacy of the drug in these tumors is unknown [4]. More troubling is that inhibiting WNT could actually make the tumors more resistant to the chemotherapy treatments. If the elevated intratumor WNT signaling is, indeed, driving the leaky vasculature and better delivery of chemotherapy, it is possible that inhibiting that intratumoral WNT signaling could improve the integrity of the blood-brain barrier and make the tumors more resistant to the chemotherapy.

\section{Sonic Hedgehog-Activated}

The Sonic hedgehog (SHH) subgroup is also named for the signaling pathway that dominates these tumors. SHH tumors have a bimodal age distribution and they afflict both the youngest and oldest patients with medulloblastoma, but spare the middle of childhood [52]. The SHH subgroup of medulloblastoma comprises $25 \%$ of all cases [73]. Overall survival for the group at large is $70 \%$ [6]. However, some key features affect outcome, specifically TP53 status. TP53-mutant cancers have a much worse prognosis with roughly a $40 \% 5$-year overall survival rate versus $80 \%$ in TP53 wild-type SHH tumors [74]. TP53 mutation status elevates the patients to high risk as these tumors are more resistant to therapy and frequently reoccur. Therefore, the WHO criteria define 2 subclasses: SHHactivated and TP53 mutant, and SHH and TP53 wild-type. In some cases, TP53 mutations have been found to be germline in medulloblastoma [74]. Li-Fraumeni syndrome, caused by germline mutation of TP53, is an inheritable or spontaneous genetic disorder that increases lifetime cancer risk, especially in pediatric cancers $[75,76]$. Because of TP53's checkpoint role for DNA damage, repair, and cell death, radiation treatment can help to accelerate the tumors and this is of keen importance in patients with Li-Fraumeni syndrome [77]. Interestingly, TP53 mutations occur in the WNT subgroups, but this does not decrease survival [74]. TP53 mutant SHH tumors also have a higher rate of MYCN locus amplification and chromothripsis [74, 78].

SHH tumors also can be linked to inheritable or spontaneous germline mutations in SHH pathway member proteinsincluding protein patched homolog 1 (PTCH1) - which cause Gorlin's syndrome [79]. Other mutations in the SHH pathway found in the tumors are the SHH receptor, smoothened (SMO), and transcription factor GLI2 [3-5]. Patients with Gorlin's syndrome have a lifetime risk of developing cancer of $>90 \%$ and radiation exacerbates this. Radiation treatment in patients with medulloblastoma with Gorlin's syndrome should be avoided as it can cause many secondary cancers such as basal cell carcinoma and meningiomas [80, 81].

The SHH subgroup itself can be further subdivided into other up to 3 subclasses using transcriptome and DNA methylation data, and it is age that is the underlying feature that stratified these subclasses [82, 83]. Infant, childhood, and adult SHH medulloblastoma have unique expression profiles and genomic lesions [83]. Overall, infant SHH has a much 
lower mutation rate than childhood and adult SHH tumors, as expected. The 2 most common mutations in infants are PTCH1 and SUFU, and nearly half of the SUFU mutations are germline events. Children aged 4 to 17 years commonly have TP53 or PTCH1 mutant cancers, and the TP53 mutations are frequently germline. Co-occurrence of GLI2 and MYCN loci amplification are also common in childhood SHH tumors. Childhood SHH medulloblastoma have a high rate of copy number aberrations, which is thought to be caused by a genome-wide shattering event known as chromothripisis. These patients have the worst outcome in the SHH subgroup, and are generally treated with high-risk protocols. Owing to the high rate of germline TP53 mutations in this subgroup, the increased use of radiation needs to be applied with caution. The WHO criteria for SHH considers TP53 status for diagnosis so this will start translating directly into the clinic as the new guideline are adopted (Table 1). Adult patients with $\mathrm{SHH}$ medulloblastoma, in contrast, are the population that may benefit most from SHH-targeted therapies as $>80 \%$ of adult $\mathrm{SHH}$ medulloblastoma are driven by either PTCH1 or SMO mutations [83]. It has been a tantalizing idea to inhibit SHH signaling as a treatment option for this subgroup, and specifically in the adult cases. SMO is a transmembrane protein that in its wild-type state is tonically inhibited by PTCH. All other elements of the SHH pathway are downstream of SMO. The best inhibitors to the SHH pathway are SMO antagonists. Mutations in proteins downstream to SMO, including intracellular activators and transcription factors, are immune to SMO-inhibiting drugs. Indeed, a recent phase II clinical trial for vismodegib in $12 \mathrm{SHH}$ subgroup medulloblastomas found that the patients who responded to the treatment were those with PTCH1 mutations [84, 85]. Vismodegib (Erivedge®) is a synthetic SMO inhibitor based on cyclopamine, the naturally occurring SMO receptor inhibitor found in Veratrum californicum (California corn lily), which famously causes cyclopia birth defects $[86,87]$. Patients with $S U F U$ and GLI1 mutations were not affected by the drug. In conclusion, SMO inhibitors act only on tumors with genetic mutations that are upstream of SMO in the pathway. Adult SHH are the best candidates to benefit from this therapy in contrast to childhood and infant patients with SHH medulloblastoma [83].

\section{Non-WNT/Non-SHH (Group 3)}

Group 3 medulloblastomas are defined by their expression of MYC, photoreceptor, and $\gamma$-aminobutyric acid-ergic gene signatures [1, 6]. Group 3 comprises close to $25 \%$ of all cases and peak diagnosis is between 3 and 5 years of age. With a $50 \%$ overall survival rate this is the deadliest subgroup of medulloblastoma. MYC signature activation is associated with poor outcome in this subgroup, and just $20 \%$ of those patients survive for 5 years $[6,7]$. MYC activation is driven by MYC loci amplification, PVT1-MYC genomic rearrangement, or another unknown mechanism [7, 88, 89]. Group 3 also commonly has isochromosome $17 \mathrm{q}$, GFII enhancer activation, and OTX2 amplification. Imbalances in chromosome 17 have been linked to poor-outcome medulloblastoma, mainly when it occurs together with $M Y C$ amplification [54, 90]. Group 3 tumors relapse via metastasis and rarely with recurrence of tumor at original location [91].

Group 3 medulloblastomas have been a major focus for experimental therapeutics because of their dismal prognosis. However, there has not been a clear path to targeting a transcription factor like MYC [92, 93]. Novel BRD4 bromodomain inhibitors such as JQ1 have been recently developed to interrupt the hyper-transcriptional activity of MYC-driven medulloblastoma cell lines and xenografts [94, 95]. As yet, there are no bromodomain inhibitors with Food and Drug Administration approval for use in adults. Currently, there are ongoing phase I clinical trials in other bromodomain inhibitors such as CPI-0610 and MK-8628 in adult cancers.

The Food and Drug Administration-approved breast cancer drug palbociclib has been recently nominated for use of MYC-driven medulloblastoma in preclinical testing using a large-cohort in silico drug screen and medulloblastoma cellline models [96]. Palbociclib is a cell-cycle checkpoint inhibitor (CDK4/6) and MYC-driven medulloblastomas appear to depend on CDK4/6 for their proliferation. Palbociclib is currently being investigated in a clinical trial for medulloblastoma and other pediatric brain cancers (ClinicalTrials.gov identifier NCT02255461).

\section{Non-WNT/Non-SHH (Group 4)}

Group 4 medulloblastomas remain the most mysterious of all. It is the most common subgroup and comprises $35 \%$ of all cases and is more likely to affect males. They were first defined by their enrichment for $\gamma$-aminobutyric acid-ergic and neuronal gene sets. Group 4 medulloblastoma have a high burden of chromosomal copy number variations. Unlike MYC-amplified group 3 tumors, isochromosome 17q does not equate to poor outcome in group 4. Patients with group 4 tumors with either i17q or chromosome 11 loss have improved outcomes compared with group 3 patients with $117 \mathrm{q}$ [97]. Group 4 medulloblastomas have an excellent prognosis, ranging from $88 \%$ to $95 \%$, depending on risk group [11]. Metastasis at is the main indicator of high risk in patients with group 4 tumors [30, 97].

Chromatin remodeler KDM6A mutations are the only subgroup-specific recurrently mutated genes and only found in $13 \%$ of patients with group 4 [98]. KDM6A is a lysine demethylase and preferentially demethylates H3K27me3 during differentiation [99, 100]. KDM6A loss of function mutations lead to a retention of these marks and a resistance of 
differentiation. Group 4 tumors without KDM6A mutations can reach a similar state by overexpressing the methylase EZH2, which puts down the H3K27me3 mark [4]. EZH2 is overexpressed in group 4 tumors via loci application, and these are mutually exclusive of the KDM6A mutations, which suggests this epigenetic inflexible state may be a more common feature of the subgroup.

\section{Future Directions}

Epigenetic mechanisms are altered across every subgroup of medulloblastoma and are emerging as a major theme across the cancer landscape following the results of large-cohort sequencing studies $[17,101]$. Because of this universality, there have been major efforts to identify treatments that target epigenetic regulators [102]. Medulloblastoma has recurrent mutations in histone modifiers [MLL2/3; KDM6A; CREBBP; histone deacetylase (HDAC)], co-repressor complexes (BCL6 corepressor, nuclear receptor corepressor 2, G protein pathway suppressor 2, LIM domain binding 1), and RNA helicases (DDX3X) [17]. Research to develop drugs that target dysregulated epigenetic mechanisms is in progress. Indeed, large-scale in vitro studies have found cancer cell lines to be sensitive drugs targeting chromatin regulators such as HDAC and enhancer of zeste homolog 2 inhibitors [103, 104]. There are many challenges, including an often narrow therapeutic window, owing to the targeting of epigenetic mechanisms in normal cells, as well as those in tumors, and a tendency for inhibition to be cytostatic rather than cytotoxic. Drug combinations that combine cytostatic and cytotoxic properties will almost certainly be needed for efficacy in cancer treatment. For example, in vitro testing of mouse-model group 3 medulloblastoma tumors has nominated a combination of antifolate drug pemetrexed and nucleoside analog gemcitabine for treatment of this devastating subgroup [105]. A similar study identified MYC-amplified group 3 tumors to be sensitive to a combination of HDAC and phosphoinositide 3-kinase inhibitors [106].

Recent studies have sought to more carefully address key prognosis questions in medulloblastoma that are outside of any one subgroup with a goal of finding minor ways to tune treatment to have the maximum benefit on outcome - both in overall survival and quality of life. Average- and high-risk medulloblastomas, for example, have been chiefly defined by the amount to residual tumor left behind from surgical resection. A large and extensive study of 787 medulloblastomas found no significant increase in survival from maximal surgical resection compared with remaining residual disease in WNT, SHH, and group 3 patients [14].

The chief cause of death in medulloblastoma is from metastatic disease. Medulloblastomas are rarely resected at recurrence, which has made study of metastatic disease difficult.
Recent studies have started to shed light on this largely unknown component of medulloblastoma. Metastatic recurrent medulloblastoma tumors have the same molecular subgroup as the primary tumors [107]. However, the genomic lesions in the metastatic tumors can be different from the primary tumor site [108]. Recurrent disseminated medulloblastoma share a common ancestor to the primary tumor but are not a direct lineage descendent of the primary tumor using molecular clock modeling of the genomic lesions in the primary and metastatic tumors samples. The metastatic tumors share some, but not all, alterations found in the primary tumor [108].

The next major strides in translating medulloblastoma genomic studies to the clinic will come from improved and rapid methods to diagnose the subgroups, and broaden the breath of preclinical in vitro and in vivo models for identifying and testing new therapeutics. Next on the horizon is how to diagnose these subgroups in a clinically tractable manner. Indeed, towards achieving this future a recent consensus article authored by the global collaborative community of pediatric neuro-oncologists behind many of these key studies outlined clinical questions that should be prioritized and proposed design of future trials [10]. Methylation status, as mentioned previously, can classify the tumor types [31]. DNA methylation status can be obtained from fixed slides of formalin-fixed paraffin-embedded tissue, which are stored and stable at room temperature for long periods of time [109, 110]. Looking to the future, this seems like the most favorable method for rapid and reliable subgroup diagnosis. Currently, clinical diagnostics are limited to copy number changes with array technologies, immunohistochemistry, and targeted sequencing [10]. However, these can only identify or rule out specific subgroups and cannot equally identify all tumor subgroups. Now solidly in the postgenomic era of medulloblastoma, we are starting to build the bridges to transfer the research findings to the clinic, and break down the major barriers that have limited this [17].

Required Author Forms Disclosure forms provided by the authors are available with the online version of this article.

Grant Support This work was supported by 2R01 CA109467, U01 CA184898, and U54 HD090255 to SLP.

\section{References}

1. Taylor MD, Northcott PA, Korshunov A, et al. Molecular subgroups of medulloblastoma: the current consensus. Acta Neuropathol 2012;123:465-472.

2. Louis DN, Perry A, Reifenberger G, et al. The 2016 World Health Organization Classification of Tumors of the Central Nervous System: a summary. Acta Neuropathol 2016;131:803-820.

3. Jones DTW, Jäger N, Kool M, et al. Dissecting the genomic complexity underlying medulloblastoma. Nature 2012;488:100-105. 
4. Robinson G, Parker M, Kranenburg TA, et al. Novel mutations target distinct subgroups of medulloblastoma. Nature 2012;488: 43-48.

5. Pugh TJ, Weeraratne SD, Archer TC, et al. Medulloblastoma exome sequencing uncovers subtype-specific somatic mutations. Nature 2012;488:106-110.

6. Cho Y-J, Tsherniak A, Tamayo P, et al. Integrative genomic analysis of medulloblastoma identifies a molecular subgroup that drives poor clinical outcome. J Clin Oncol 2011;29:1424-1430.

7. Tamayo P, Cho Y-J, Tsherniak A, et al. Predicting relapse in patients with medulloblastoma by integrating evidence from clinical and genomic features. J Clin Oncol 2011;29:1415-1423.

8. Fernandez-Teijeiro A, Betensky RA, Sturla LM, Kim JYH, Tamayo P, Pomeroy SL. Combining gene expression profiles and clinical parameters for risk stratification in medulloblastomas. J Clin Oncol 2004;22:994-998.

9. Pomeroy SL, Tamayo P, Gaasenbeek M, et al. Prediction of central nervous system embryonal tumour outcome based on gene expression. Nature 2002;415:436-442.

10. Ramaswamy V, Remke M, Bouffet E, et al. Risk stratification of childhood medulloblastoma in the molecular era: the current consensus. Acta Neuropathol 2016;131:821-831.

11. Ramaswamy V, Remke M, Adamski J, et al. Medulloblastoma subgroup-specific outcomes in irradiated children: who are the true high-risk patients? Neuro Oncol 2016;18:291-297.

12. Bueren von AO, Kortmann R-D, Hoff von K, et al. Treatment of children and adolescents with metastatic medulloblastoma and prognostic relevance of clinical and biologic parameters. J Clin Oncol 2016;34:4151-4160.

13. Moxon-Emre I, Taylor MD, Bouffet E, et al. Intellectual outcome in molecular subgroups of medulloblastoma. J Clin Oncol 2016;34:4161-4170.

14. Thompson EM, Hielscher T, Bouffet E, et al. Prognostic value of medulloblastoma extent of resection after accounting for molecular subgroup: a retrospective integrated clinical and molecular analysis. Lancet Oncol 2016;17:484-495.

15. Lawrence MS, Stojanov P, Mermel CH, et al. Discovery and saturation analysis of cancer genes across 21 tumour types. Nature 2014;505:495-501.

16. Lawrence MS, Stojanov P, Polak P, et al. Mutational heterogeneity in cancer and the search for new cancer-associated genes. Nature 2013;499:214-218.

17. Archer TC, Pomeroy SL. Medulloblastoma biology in the postgenomic era. Future Oncol 2012;8:1597-1604.

18. Armstrong GL, Conn LA, Pinner RW. Trends in infectious disease mortality in the United States during the 20th century. JAMA 1999;281:61-66.

19. Thariat J, Hannoun-Levi J-M, Sun Myint A, Vuong T, Gérard J-P. Past, present, and future of radiotherapy for the benefit of patients. Nat Rev Clin Oncol 2013;10:52-60.

20. Higgins KA, O'Connell K, Liu Y, et al. National Cancer Database analysis of proton versus photon radiation therapy in non-small cell lung cancer. Int J Radiat Oncol Biol Phys 2017;97:128-137.

21. Emmons MF, Faião-Flores F, Smalley KSM. The role of phenotypic plasticity in the escape of cancer cells from targeted therapy. Biochem Pharmacol 2016;122:1-9.

22. Ades F, Metzger-Filho O. Targeting the cellular signaling: BRAF inhibition and beyond for the treatment of metastatic malignant melanoma. Dermatol Res Pract 2012;2012:259170.

23. Nazarian R, Shi H, Wang Q, et al. Melanomas acquire resistance to B-RAF(V600E) inhibition by RTK or N-RAS upregulation. Nature 2010;468:973-977.

24. Gambacorti-Passerini C, Antolini L, Mahon F-X, et al. Multicenter independent assessment of outcomes in chronic myeloid leukemia patients treated with imatinib. J Natl Cancer Inst 2011;103:553-561.
25. Carroll M, Ohno-Jones S, Tamura S, et al. CGP 57148, a tyrosine kinase inhibitor, inhibits the growth of cells expressing BCRABL, TEL-ABL, and TEL-PDGFR fusion proteins. Blood 1997;90:4947-4952.

26. Radich JP, Kopecky KJ, Appelbaum FR, et al. A randomized trial of dasatinib $100 \mathrm{mg}$ versus imatinib $400 \mathrm{mg}$ in newly diagnosed chronic-phase chronic myeloid leukemia. Blood 2012;120:3898 3905.

27. Czerwionka M, Korf HW, Hoffmann O, Busch H, Schachenmayr W. Differentiation in medulloblastomas: correlation between the immunocytochemical demonstration of photoreceptor markers (Santigen, rod-opsin) and the survival rate in 66 patients. Acta Neuropathol 1989;78:629-636.

28. Schofield D, West DC, Anthony DC, Marshal R, Sklar J. Correlation of loss of heterozygosity at chromosome $9 \mathrm{q}$ with histological subtype in medulloblastomas. Am J Pathol 1995;146: 472-480.

29. Northcott PA, Shih DJH, Remke M, et al. Rapid, reliable, and reproducible molecular sub-grouping of clinical medulloblastoma samples. Acta Neuropathol 2012;123:615-626.

30. Kool M, Korshunov A, Remke M, et al. Molecular subgroups of medulloblastoma: an international meta-analysis of transcriptome, genetic aberrations, and clinical data of WNT, SHH, Group 3, and Group 4 medulloblastomas. Acta Neuropathol 2012;123:473484.

31. Hovestadt V, Jones DTW, Picelli S, et al. Decoding the regulatory landscape of medulloblastoma using DNA methylation sequencing. Nature 2014;510:537-541.

32. Mueller S, Chang S. Pediatric brain tumors: current treatment strategies and future therapeutic approaches. Neurotherapeutics 2009;6:570-586.

33. Packer RJ, Gajjar A, Vezina G, et al. Phase III study of craniospinal radiation therapy followed by adjuvant chemotherapy for newly diagnosed average-risk medulloblastoma. J Clin Oncol 2006;24:4202-4208.

34. Merchant TE, Kun LE, Krasin MJ, et al. Multi-institution prospective trial of reduced-dose craniospinal irradiation (23.4 Gy) followed by conformal posterior fossa (36 Gy) and primary site irradiation $(55.8 \mathrm{~Gy})$ and dose-intensive chemotherapy for average-risk medulloblastoma. Int J Radiat Oncol Biol Phys 2008;70:782-787.

35. Gajjar A, Chintagumpala M, Ashley D, et al. Risk-adapted craniospinal radiotherapy followed by high-dose chemotherapy and stem-cell rescue in children with newly diagnosed medulloblastoma (St Jude Medulloblastoma-96): long-term results from a prospective, multicentre trial. Lancet Oncol 2006;7:813-820.

36. Wahba HA, Abu-Hegazy M, Wasel Y, Ismail EI, Zidan AS. Adjuvant chemotherapy after reduced craniospinal irradiation dose in children with average-risk medulloblastoma: a 5-year follow-up study. J BUON 2013;18:425-429.

37. Packer RJ, Goldwein J, Nicholson HS, et al. Treatment of children with medulloblastomas with reduced-dose craniospinal radiation therapy and adjuvant chemotherapy: a Children's Cancer Group Study. J Clin Oncol 1999;17:2127-2136.

38. Lafay-Cousin L, Smith A, Chi SN, et al. Clinical, pathological, and molecular characterization of infant medulloblastomas treated with sequential high-dose chemotherapy. Pediatr Blood Cancer 2016;63:1527-1534.

39. Tarbell NJ, Friedman H, Polkinghorn WR, et al. High-risk medulloblastoma: a pediatric oncology group randomized trial of chemotherapy before or after radiation therapy (POG 9031). J Clin Oncol 2013;31:2936-2941.

40. Tamburrini G, Frassanito P, Chieffo D, Massimi L, Caldarelli M, Di Rocco C. Cerebellar mutism. Childs Nerv Syst 2015;31:18411851. 
41. Ris MD, Packer R, Goldwein J, Jones-Wallace D, Boyett JM. Intellectual outcome after reduced-dose radiation therapy plus adjuvant chemotherapy for medulloblastoma: a Children's Cancer Group study. J Clin Oncol 2001;19:3470-3476.

42. Chi SN, Zimmerman MA, Yao X, et al. Intensive multimodality treatment for children with newly diagnosed CNS atypical teratoid rhabdoid tumor. J Clin Oncol 2009;27:385-389.

43. Goldwein JW, Radcliffe J, Johnson J, et al. Updated results of a pilot study of low dose craniospinal irradiation plus chemotherapy for children under five with cerebellar primitive neuroectodermal tumors (medulloblastoma). Int J Radiat Oncol Biol Phys 1996;34: 899-904.

44. Walker MD, Green SB, Byar DP, et al. Randomized comparisons of radiotherapy and nitrosoureas for the treatment of malignant glioma after surgery. N Engl J Med 1980;303:1323-1329.

45. Merchant TE, Kiehna EN, Li C, et al. Modeling radiation dosimetry to predict cognitive outcomes in pediatric patients with CNS embryonal tumors including medulloblastoma. Int J Radiat Oncol Biol Phys 2006;65:210-221.

46. Barrera M, Shaw AK, Speechley KN, Maunsell E, Pogany L. Educational and social late effects of childhood cancer and related clinical, personal, and familial characteristics. Cancer 2005;104: 1751-1760.

47. Palmer SL, Gajjar A, Reddick WE, et al. Predicting intellectual outcome among children treated with 35-40 Gy craniospinal irradiation for medulloblastoma. Neuropsychology 2003;17:548555.

48. Bansal LR, Belair J, Cummings D, Zuccoli G. Late-onset radiation-induced vasculopathy and stroke in a child with medulloblastoma. J Child Neurol 2015;30:800-802.

49. Ullrich NJ, Pomeroy SL. Molecular genetics of pediatric central nervous system tumors. Curr Oncol Rep 2006;8:423-429.

50. Neglia JP, Robison LL, Stovall M, et al. New primary neoplasms of the central nervous system in survivors of childhood cancer: a report from the Childhood Cancer Survivor Study. J Natl Cancer Inst 2006;98:1528-1537.

51. Benson PJ, Sung JH. Cerebral aneurysms following radiotherapy for medulloblastoma. J Neurosurg 1989;70:545-550.

52. Northcott PA, Korshunov A, Witt H, et al. Medulloblastoma comprises four distinct molecular variants. J Clin Oncol 2011;29: 1408-1414.

53. Ellison DW, Dalton J, Kocak M, et al. Medulloblastoma: clinicopathological correlates of SHH, WNT, and non-SHH/WNT molecular subgroups. Acta Neuropathol 2011;121:381-396.

54. Clifford SC, Lannering B, Schwalbe EC, et al. Biomarker-driven stratification of disease-risk in non-metastatic medulloblastoma: results from the multi-center HIT-SIOP-PNET4 clinical trial. Oncotarget 2015;6:38827-38839.

55. Bueno R, Stawiski EW, Goldstein LD, et al. Comprehensive genomic analysis of malignant pleural mesothelioma identifies recurrent mutations, gene fusions and splicing alterations. Nat Genet 2016;48:407-416.

56. Seiwert TY, Zuo Z, Keck MK, et al. Integrative and comparative genomic analysis of HPV-positive and HPV-negative head and neck squamous cell carcinomas. Clin Cancer Res 2014;21:632641.

57. Jiang L, Gu Z-H, Yan Z-X, et al. Exome sequencing identifies somatic mutations of DDX3X in natural killer/T-cell lymphoma. Nat Genet 2015;47:1061-1066.

58. Ojha J, Secreto CR, Rabe KG, et al. Identification of recurrent truncated DDX3X mutations in chronic lymphocytic leukaemia. Br J Haematol 2015;169:445-448.

59. Wang K, Kan J, Yuen ST, et al. Exome sequencing identifies frequent mutation of ARID1A in molecular subtypes of gastric cancer. Nat Genet 2011;43:1219-1223.
60. Dunford A, Weinstock DM, Savova V, et al. Tumor-suppressor genes that escape from $\mathrm{X}$-inactivation contribute to cancer sex bias. Nat Genet 2016;49:10-16.

61. Cheng F, Liu C, Lin C-C, et al. A gene gravity model for the evolution of cancer genomes: a study of 3,000 cancer genomes across 9 cancer types. PLOS Comput Biol 2015;11:e1004497.

62. Trubicka J, Szperl M, Grajkowska W, et al. Identification of a novel inherited ALK variant M1199L in the WNT type of medulloblastoma. Folia Neuropathol 2016;54:23-30.

63. Coco S, De Mariano M, Valdora F, et al. Identification of ALK germline mutation (3605delG) in pediatric anaplastic medulloblastoma. J Hum Genet 2012;57:682-684.

64. Horie R, Watanabe M, Ishida T, et al. The NPM-ALK oncoprotein abrogates CD30 signaling and constitutive NF-kappaB activation in anaplastic large cell lymphoma. Cancer Cell 2004;5:353-364.

65. Ma B, Hottiger MO. Crosstalk between Wnt $/ \beta$-catenin and NF$\mathrm{KB}$ signaling pathway during inflammation. Front Immunol 2016;7:378.

66. Hamilton SR, Liu B, Parsons RE, et al. The molecular basis of Turcot's syndrome. N Engl J Med 1995;332:839-847.

67. Morin PJ, Sparks AB, Korinek V, et al. Activation of beta-cateninTcf signaling in colon cancer by mutations in beta-catenin or APC. Science 1997;275:1787-1790.

68. Huang H, Mahler-Araujo BM, Sankila A, et al. APC mutations in sporadic medulloblastomas. Am J Pathol 2000;156:433-437.

69. Phoenix TN, Patmore DM, Boop S, et al. Medulloblastoma genotype dictates blood brain barrier phenotype. Cancer Cell 2016;29: 508-522.

70. Daneman R, Agalliu D, Zhou L, Kuhnert F, Kuo CJ, Barres BA. Wnt/beta-catenin signaling is required for CNS, but not non-CNS, angiogenesis. Proc Natl Acad Sci U S A 2009;106:641-646.

71. Kahn M. Can we safely target the WNT pathway? Nat Rev Drug Discov 2014;13:513-532.

72. Dey N, Barwick BG, Moreno CS, et al. Wnt signaling in triple negative breast cancer is associated with metastasis. BMC Cancer 2013; 13:537.

73. Northcott PA, Korshunov A, Pfister SM, Taylor MD. The clinical implications of medulloblastoma subgroups. Nat Rev Neurol 2012;8:340-351.

74. Zhukova N, Ramaswamy V, Remke M, et al. Subgroup-specific prognostic implications of TP53 mutation in medulloblastoma. J Clin Oncol 2013;31:2927-2935.

75. Kappel S, Janschek E, Wolf B, et al. TP53 germline mutation may affect response to anticancer treatments: analysis of an intensively treated Li-Fraumeni family. Breast Cancer Res Treat 2015;151: 671-678.

76. Malkin D, Friend SH, Li FP, Strong LC. Germ-line mutations of the p53 tumor-suppressor gene in children and young adults with second malignant neoplasms. N Engl J Med 1997;336:734.

77. Tchelebi L, Ashamalla H, Graves PR. Mutant p53 and the response to chemotherapy and radiation. Subcell Biochem 2014;85:133-159.

78. Rausch T, Jones DTW, Zapatka M, et al. Genome sequencing of pediatric medulloblastoma links catastrophic DNA rearrangements with TP53 mutations. Cell 2012;148:59-71.

79. Smith MJ, Beetz C, Williams SG, et al. Germline mutations in SUFU cause Gorlin syndrome-associated childhood medulloblastoma and redefine the risk associated with PTCH1 mutations. J Clin Oncol 2014;32:4155-4161.

80. Thalakoti S, Geller T. Basal cell nevus syndrome or Gorlin syndrome. Handb Clin Neurol 2015;132:119-128.

81. O'Malley S, Weitman D, Olding M, Sekhar L. Multiple neoplasms following craniospinal irradiation for medulloblastoma in a patient with nevoid basal cell carcinoma syndrome. Case report. J Neurosurg 1997;86:286-288. 
82. Northcott PA, Hielscher T, Dubuc A, et al. Pediatric and adult sonic hedgehog medulloblastomas are clinically and molecularly distinct. Acta Neuropathol 2011;122:231-240.

83. Kool M, Jones DTW, Jäger N, et al. Genome sequencing of SHH medulloblastoma predicts genotype-related response to smoothened inhibition. Cancer Cell 2014;25:393-405.

84. Ransohoff KJ, Sarin KY, Tang JY. Smoothened inhibitors in Sonic hedgehog subgroup medulloblastoma. J Clin Oncol 2015;33: 2692-2694.

85. Robinson GW, Orr BA, Wu G, et al. Vismodegib exerts targeted efficacy against recurrent sonic hedgehog-subgroup medulloblastoma: results from phase II Pediatric Brain Tumor Consortium Studies PBTC-025B and PBTC-032. J Clin Oncol 2015;33: 2646-2654.

86. Chen JK, Taipale J, Cooper MK, Beachy PA. Inhibition of Hedgehog signaling by direct binding of cyclopamine to Smoothened. Genes Dev 2002;16:2743-2748.

87. Taipale J, Chen JK, Cooper MK, et al. Effects of oncogenic mutations in Smoothened and Patched can be reversed by cyclopamine. Nature 2000;406:1005-1009.

88. Ryan SL, Schwalbe EC, Cole M, et al. MYC family amplification and clinical risk-factors interact to predict an extremely poor prognosis in childhood medulloblastoma. Acta Neuropathol 2012;123: 501-513.

89. Northcott PA, Shih DJH, Peacock J, et al. Subgroup-specific structural variation across 1,000 medulloblastoma genomes. Nature 2012;488:49-56.

90. Zhukova N, Ramaswamy V, Remke M, et al. WNT activation by lithium abrogates TP53 mutation associated radiation resistance in medulloblastoma. Acta Neuropathol Commun 2014;2:174.

91. Ramaswamy V, Remke M, Bouffet E, et al. Recurrence patterns across medulloblastoma subgroups: an integrated clinical and molecular analysis. Lancet Oncol 2013;14:1200-1207.

92. Andrieu G, Belkina AC, Denis GV. Clinical trials for BET inhibitors run ahead of the science. Drug Discov Today Technol 2016;19:45-50.

93. Jung M, Gelato KA, Fernández-Montalván A, Siegel S, Haendler B. Targeting BET bromodomains for cancer treatment. Epigenomics. 2015;7:487-501.

94. Bandopadhayay P, Bergthold G, Nguyen B, et al. BET bromodomain inhibition of MYC-amplified medulloblastoma. Clin Cancer Res 2014;20:912-925.

95. Venkataraman S, Alimova I, Balakrishnan I, et al. Inhibition of BRD4 attenuates tumor cell self-renewal and suppresses stem cell signaling in MYC driven medulloblastoma. Oncotarget 2014;5: $2355-2371$.
96. Hanaford AR, Archer TC, Price A, et al. DiSCoVERing innovative therapies for rare tumors: combining genetically accurate disease models with in silico analysis to identify novel therapeutic targets. Clin Cancer Res 2016;22:3903-3914.

97. Shih DJH, Northcott PA, Remke M, et al. Cytogenetic prognostication within medulloblastoma subgroups. J Clin Oncol 2014;32: 886-896.

98. Northcott PA, Jones DTW, Kool M, et al. Medulloblastomics: the end of the beginning. Nat Rev Cancer 2012;12:818-834.

99. Agger K, Cloos PAC, Christensen J, et al. UTX and JMJD3 are histone H3K27 demethylases involved in HOX gene regulation and development. Nature 2007;449:731-734.

100. Wang JK, Tsai M-C, Poulin G, et al. The histone demethylase UTX enables RB-dependent cell fate control. Genes Dev 2010;24:327-332.

101. You JS, Jones PA. Cancer genetics and epigenetics: two sides of the same coin? Cancer Cell 2012;22:9-20.

102. Pfister SX, Ashworth A. Marked for death: targeting epigenetic changes in cancer. Nat Rev Drug Discov 2017 Mar 10 [Epub ahead of print].

103. Cohen AL, Piccolo SR, Cheng L, et al. Genomic pathway analysis reveals that EZH2 and HDAC4 represent mutually exclusive epigenetic pathways across human cancers. BMC Med Genomics 2013;6:35.

104. Wang K, Shrestha R, Wyatt AW, et al. A meta-analysis approach for characterizing pan-cancer mechanisms of drug sensitivity in cell lines. PLOS ONE. 2014;9:e103050.

105. Morfouace M, Shelat A, Jacus M, et al. Pemetrexed and gemcitabine as combination therapy for the treatment of Group3 medulloblastoma. Cancer Cell 2014;25:516-529.

106. Pei Y, Liu K-W, Wang J, et al. HDAC and PI3K antagonists cooperate to inhibit growth of MYC-driven medulloblastoma. Cancer Cell 2016;29:311-323.

107. Wang X, Dubuc AM, Ramaswamy V, et al. Medulloblastoma subgroups remain stable across primary and metastatic compartments. Acta Neuropathol 2015;129:449-457.

108. Morrissy AS, Garzia L, Shih DJH, et al. Divergent clonal selection dominates medulloblastoma at recurrence. Nature 2016;529:35137.

109. Archer TC, Pomeroy SL. Defining the molecular landscape of ependymomas. Cancer Cell 2015;27:613-615.

110. Pajtler KW, Witt H, Sill M, et al. Molecular Classification of ependymal tumors across all CNS compartments, histopathological grades, and age groups. Cancer Cell 2015;27: 728-743. 